\title{
Reducing Unnecessary Treatment of Asymptomatic Elevated Blood Pressure with Intravenous Medications on the General Internal Medicine Wards: A Quality Improvement Initiative
}

\author{
Zachary G Jacobs, MD ${ }^{1,2 *}$; Nader Najafi, MD'; Margaret C Fang, MD'; Priya A Prasad, PhD, MPH'; \\ Yumiko Abe-Jones, MS'; Andrew D Auerbach, MD, MPH, SFHM'1; Sajan Patel, MD ${ }^{1 *}$
}

${ }^{1}$ Division of Hospital Medicine, Department of Medicine, University of California, San Francisco, San Francisco, California; ${ }^{2}$ Dr. Jacobs is now with Division of Hospital Medicine, Oregon Health \& Science University, Portland, Oregon.

BACKGROUND: Asymptomatic elevated blood pressure $(B P)$ is common in the hospital. There is no evidence supporting the use of intravenous (IV) antihypertensives in this setting.

OBJECTIVE: To determine the prevalence and effects of treating asymptomatic elevated BP with IV antihypertensives and to investigate the efficacy of a quality improvement (QI) initiative aimed at reducing utilization of these medications.

DESIGN: Retrospective cohort study.

SETTING: Urban academic hospital.

PATIENTS: Patients admitted to the general medicine service, including the intensive care unit (ICU), with $\geq 1$ episode of asymptomatic elevated BP (>160/90 mm Hg) during hospitalization.

INTERVENTION: A two-tiered, Ol initiative.

MEASUREMENTS: The primary outcome was the monthly proportion of patients with asymptomatic elevated BP treated with IV labetalol or hydralazine. We also analyzed median BP and rates of balancing outcomes (ICU transfers, rapid responses, cardiopulmonary arrests).

RESULTS: We identified 2,306 patients with $\geq 1$ episode of asymptomatic elevated BP during the 10-month preintervention period, of which 251 (11\%) received IV antihypertensives. In the four-month postintervention period, 70 of 934 (7\%) were treated. The odds of being treated were $38 \%$ lower in the postintervention period after adjustment for baseline characteristics, including length of stay and illness severity $(\mathrm{OR}=0.62 ; 95 \% \mathrm{Cl} 0.47$ $0.83 ; P=.001)$. Median SBP was similar between pre- and postintervention (167 vs $168 \mathrm{~mm} \mathrm{Hg} P=.78$ ), as were the adjusted proportions of balancing outcomes.

CONCLUSIONS: Hospitalized patients with asymptomatic elevated BP are commonly treated with IV antihypertensives, despite the lack of evidence. A QI initiative was successful at reducing utilization of these medications. Journal of Hospital Medicine 2019;14:144150. (C) 2019 Society of Hospital Medicine

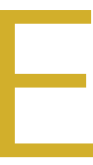

levated blood pressure (BP) is common among hospitalized adults, with prevalence estimates between $50 \%$ and 70\%.' Many factors can cause or exacerbate BP elevations in the setting of acute illness, such as pain, anxiety, medication withdrawal, and volume status, among others. ${ }^{2}$ While there are clear evidence-based recommendations for treating hypertension (HTN) in the ambulatory setting, ${ }^{3}$ guidelines for the management of elevated BP in the hospital are lacking. ${ }^{4,5}$

Hypertensive crises are generally recognized as warranting rapid reduction in $\mathrm{BP}^{; 6-8}$ however, these represent the minority of cases. ${ }^{9,10}$ Far more common in the hospital are patients with asymptomatic elevated BP, a population for which there is no

\footnotetext{
*Corresponding Authors: (1) Zachary G. Jacobs, MD: E-mail: JacobsZ@ohsu edu. (2) Sajan Patel, MD; E-mail: Sajan.Patel@ucsf.edu

Additional Supporting Information may be found in the online version of this article.
}

Received: May 31, 2018; Revised: August 3, 2018; Accepted: August 13, 2018

(c) 2019 Society of Hospital Medicine DOI 10.12788/jhm.3087 high-quality evidence and no guidelines supporting the use of intravenous (IV) antihypertensives. ${ }^{11,12}$ Treatment with such medications has been associated with highly variable clinical responses $^{13-15}$ and may result in adverse events, such as hypotension. ${ }^{10}$

To date, only a small number of studies have investigated the treatment of asymptomatic elevated BP among hospitalized adults. ${ }^{10,13-15}$ These have suggested that IV antihypertensives are utilized frequently in this setting, often for only modestly elevated BPs; however, the studies have tended to be small, not racially diverse, and limited to noncritically ill patients. Furthermore, while it is generally accepted that reducing the use of IV antihypertensives among asymptomatic patients would have no adverse impact, to our knowledge there have been no published studies which have instituted such an initiative while measuring balancing outcomes.

The purpose of this study was to further the existing literature by defining the prevalence and effects of IV antihypertensive medication utilization among a medically complex, multiracial population of asymptomatic medical inpatients using a large electronic dataset and to evaluate the impact of a division-wide, 
two-tiered quality improvement (QI) initiative on the rates of IV antihypertensive utilization and patient outcomes.

\section{METHODS}

\section{Setting}

The study was conducted at the University of California, San Francisco (UCSF), an 800-bed tertiary care, academic medical center. It was approved by the UCSF Institutional Review Board. General medicine patients at UCSF are distributed between teaching and direct-care (hospitalist) services. The intensive care unit (ICU) is "open," meaning the medicine service acts as the primary team for all nonsurgical ICU patients. This study included all adult general medicine patients admitted to UCSF Medical Center between January 1, 2017 and March 1, 2018, including those in the ICU.

\section{Study Population and Data Collection}

The UCSF Medical Center uses the electronic health record (EHR) Epic (Epic 2017, Epic Systems Corporation, Verona, Wisconsin) for all clinical care. We obtained computerized EHR data from Clarity, the relational database that stores Epic's inpatient data in thousands of tables, including orders, medications, laboratory and radiology results, vital signs, patient demographics, and notes. We identified all adult patients hospitalized on the general medicine service with $\geq 1$ episode of elevated BP (>160/90 $\mathrm{mm} \mathrm{Hg}$ ) at any point during their hospitalization who were not on a vasopressor medication at the time of the vital sign recording.

We further identified all instances in which either IV labetalol or hydralazine were administered to these patients. These two agents were chosen because they are the only IV antihypertensives used commonly at our institution for the treatment of asymptomatic elevated BP among internal medicine patients. Only those orders placed by a general medicine provider or reconciled by a general medicine provider upon transfer from another service were included. For each medication administration timestamp, we collected vital signs before and after the administration, along with the ordering provider and the clinical indication that was documented in the electronic order. To determine if a medication was administered with concern for end-organ injury, we also extracted orders that could serve as a proxy for the provider's clinical assessment-namely electrocardiograms, serum troponins, chest x-rays, and computerized tomography scans of the head-which were placed in the one hour preceding or 15 minutes following administration of an IV antihypertensive medication.

To assess for comorbid conditions, including a preexisting diagnosis of HTN, we collected International Classification of Diseases (ICD)-9/10 diagnosis codes. Further, we also extracted All Patient Refined Diagnosis-Related Group (APR-DRG) weights, which are a standardized measure of illness severity based on relative resource consumption during hospitalization. 16,17

Patients were categorized as having either "symptomatic" or "asymptomatic" elevated BP. We defined symptomatic elevated BP as having received treatment with an IV medication with provider concern for end-organ injury, as defined above.
We further identified all patients in which tight BP control may be clinically indicated on the basis of the presence of any of the following ICD-9/10 diagnosis codes at the time of hospital discharge: myocardial infarction, ischemic stroke, intracranial hemorrhage, subarachnoid hemorrhage, subdural hematoma, aortic dissection, hypertensive emergency, or hypertensive encephalopathy. All patients with symptomatic elevated BP or any of the above ICD-9/10 diagnoses were excluded from the analysis, since administration of IV antihypertensive medications would plausibly be warranted in these clinical scenarios.

The encounter numbers from the dataset were used to link to patient demographic data, which included age, sex, race, ethnicity, primary language, and insurance status. Finally, we identified all instances of rapid response calls, ICU transfers, and code blues (cardiopulmonary arrests) for each patient in the dataset.

\section{Blood Pressure Measurements}

BP data were collected from invasive BP (IBP) monitoring devices and noninvasive BP cuffs. For patients with BP measurements recorded concomitantly from both IBP (ie, arterial lines) in addition to noninvasive BP cuffs, the arterial line reading was favored. All systolic BP (SBP) readings $>240 \mathrm{~mm} \mathrm{Hg}$ from arterial lines were excluded, as this has previously been described as the upper physiologic limit for IBP readings. ${ }^{18}$

\section{Primary Outcome}

The primary outcome for the study was the proportion of patients treated with IV antihypertensive medications (labetalol or hydralazine). Using aggregate data, we calculated the number of patients who were treated at least once with an IV antihypertensive in a given month (numerator), divided by the number of patients with $\geq 1$ episode of asymptomatic elevated BP that month (denominator). The denominator was considered to be the population of patients "at risk" of being treated with IV antihypertensive medications. For patients with multiple admissions during the study period, each admission was considered separately. These results are displayed in the upper portion of the run chart (Figure).

\section{Secondary Outcomes}

To investigate blood pressure trends over time, we analyzed BP in three ways. First, we analyzed the median SBP for the entire population. Second, to determine clinical responses to IV antihypertensive medications among patients receiving treatment, we calculated the population medians for the pretreatment SBP, the change in SBP from pretreatment baseline, and the posttreatment SBP. Third, we calculated the average median SBP on a monthly basis for the duration of the study. This was achieved by calculating the median value of all SBPs for an individual patient, then averaging across all patients in a given month. The average monthly median SBPs are displayed in the lower portion of the Figure.

To investigate whether the intervention was associated with negative patient outcomes, the proportions of several balancing outcomes were compared between pre- and postinterven- 


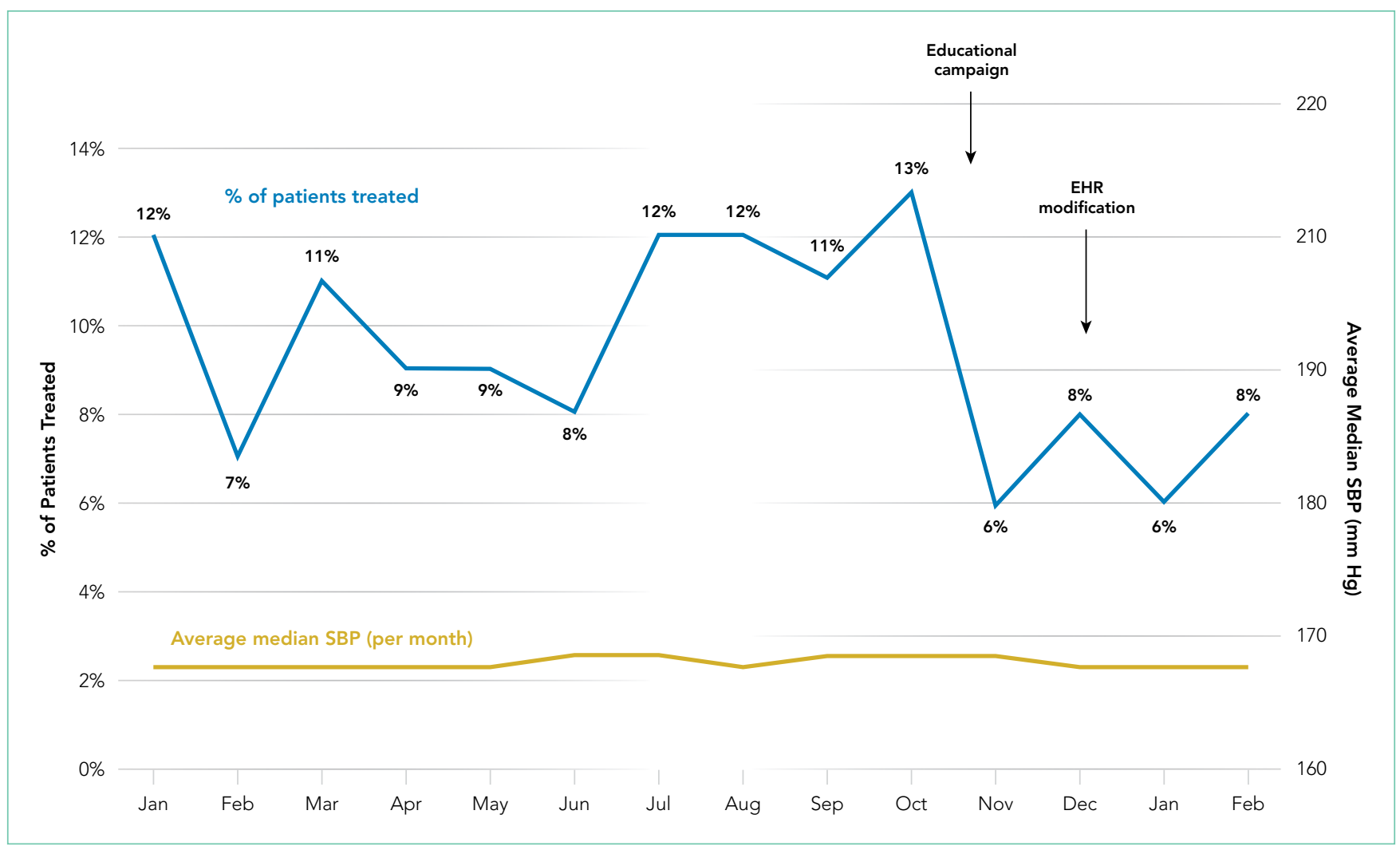

FIG. Percentage of patients with asymptomatic elevated blood pressure receiving intravenous antihypertensive medications, and average median SBP, per month.

Abbreviations: EHR, electronic health record; SBP, systolic blood pressure.

tion periods, including ICU transfers, rapid response calls, and code blues (cardiopulmonary arrests).

Development and Implementation of an Intervention to Reduce Excessive IV Antihypertensive Use After establishing the baseline prevalence of IV antihypertensive medication use at our institution, we developed a Ql initiative with the goal of reducing IV antihypertensive medication utilization by the general medicine service for the treatment of asymptomatic patients. We hypothesized that potential contributors to overutilization might include lack of education, provider/nursing discomfort, and a system designed to mandate provider notification for even modestly elevated BPs. The Ql initiative, which took place between October 2017 and December 2017, was designed to address these potential contributors and was comprised of a division-wide, two-tiered, bundled intervention. Our choice of a two-tiered approach was based on the fact that successful culture change is challenging, along with the existing evidence that multifaceted Ql interventions are more often successful than single-tiered approaches. ${ }^{19}$

The first tier of the initiative included an educational campaign referred to colloquially as "NoIVForHighBP," which targeted residents, hospitalists, and nursing staff. The campaign consisted of a series of presentations, best practice updates, handouts, and posters displayed prominently in shared workspaces. The educational content focused on alternative ap- proaches to the management of asymptomatic elevated BP in the hospital, such as identification and treatment of pain, anxiety, volume overload, or other contributing factors (see supplemental materials). These educational outreaches occurred periodically between October 4, 2017 and November 20, 2017, with the bulk of the educational efforts taking place during November. Therefore, November 1, 2017 was designated the start date for the intervention period.

The second tier of the intervention included the liberalization of the EHR BP notification parameters on the standard inpatient admission order set from $>160 / 90 \mathrm{~mm} \mathrm{Hg}$ to $>180 / 90$ $\mathrm{mm} \mathrm{Hg}$. This change took effect on 12/6/2017. The decision to modify the BP notification parameters was based on the hypothesis that mandatory notifications for modestly elevated BPs may prompt providers to reflexively order IV antihypertensive medications, especially during times of cross-coverage or high clinical workload.

\section{Statistical Analysis}

All statistical analyses were performed using Stata software version 15 (StataCorp. 2017. Stata Statistical Software: Release 15. College Station, Texas: StataCorp LLC). Baseline patient characteristics were compared using nonparametric tests of significance. Population median SBPs were compared between pre- and postintervention periods using Mood's Median Test, which was selected because the data were distributed nonnormally, and variances between samples were unequal. 
TABLE 1. Comparison of Baseline Patient Characteristics between Pre- and Postintervention Periods

\begin{tabular}{|c|c|c|c|}
\hline & $\begin{array}{l}\text { Preintervention, } \\
n=2,306\end{array}$ & $\begin{array}{l}\text { Postintervention, } \\
\qquad \mathrm{n}=934\end{array}$ & $P$ Value \\
\hline Age, median (IQR) & $67(55-80)$ & $69(57-83)$ & .01 \\
\hline Sex, n (\%) & & & .09 \\
\hline Male & $1,186(51)$ & 450 (48) & \\
\hline Female & $1,120(49)$ & $485(52)$ & \\
\hline Race, n (\%) & & & .22 \\
\hline White & $917(40)$ & $372(40)$ & \\
\hline Black & $442(19)$ & $149(16)$ & \\
\hline Asian & $523(23)$ & $237(25)$ & \\
\hline Hispanic & $216(9)$ & $88(9)$ & \\
\hline Other & $199(9)$ & $88(9)$ & \\
\hline \multicolumn{4}{|l|}{ Primary language, $n(\%)$} \\
\hline Non-English & $475(21)$ & $222(24)$ & .05 \\
\hline Insurance, $n(\%)$ & & & .09 \\
\hline Commercial & $314(14)$ & $119(12)$ & \\
\hline Medicaid & $532(23)$ & $184(20)$ & \\
\hline Medicare & $1,439(62)$ & $625(67)$ & \\
\hline Other ${ }^{\mathrm{a}}$ & $21(0.9)$ & $6(0.6)$ & \\
\hline Hypertension present on admission, $n(\%)$ & $1,732(75)$ & $659(71)$ & .01 \\
\hline APR-DRG Weight, median (IQR) & $1.34(0.99-1.77)$ & $1.48(1.00-1.82)$ & $<.001$ \\
\hline Inpatient length of stay, median (IQR) & $4.6(2.8-8.0)$ & $5.1(2.9-9.2)$ & .004 \\
\hline $\begin{array}{l}\text { 'Includes uninsured, workers' compensati } \\
\text { Abbreviations: APR-DRG, all patient refine }\end{array}$ & & & \\
\hline
\end{tabular}

Among patients treated with IV antihypertensive medications, we compared the proportion of pretreatment SBPs falling into each of three specified ranges (SBP $<180 \mathrm{~mm} \mathrm{Hg}$, SBP 180-199 $\mathrm{mm} \mathrm{Hg}$, and SBP >200 mm Hg) between baseline and intervention periods using chi-squared tests.

Using aggregate data, we compared the unadjusted proportion of patients treated with IV antihypertensive medications between pre- and postintervention periods using a chi-squared test. Next, using patient-level data, a logistic regression analysis was performed to examine the association between receipt of IV antihypertensive medications and time (dichotomized between pre- and postintervention periods) while adjusting for age, sex, race, ethnicity, primary language, insurance status, preexisting HTN, length of stay, and APR-DRG weight.

Rates of balancing outcomes were compared using chisquared tests. A logistic regression analysis using patient-level data was also performed to investigate the association between each of these outcomes and the intervention period (pre vs post) while adjusting for age, sex, race, ethnicity, primary language, insurance status, preexisting HTN, length of stay, and APR-DRG weight.

\section{RESULTS}

\section{Baseline Period}

We identified 2,306 patients with $\geq 1$ episode of asymptomatic elevated BP during the 10-month preintervention period. Patients on average experienced 9 episodes of elevated BP per hospitalization, representing 21,207 potential opportunities for treatment. Baseline characteristics are summarized in Table 1. In general, this represents an older population that was medically complex and multiracial.

Of these patients, 251 (11\%) received IV hydralazine and/or labetalol at least once during their hospitalization, with a total of 597 doses administered. Among those treated, a median of 2 doses were given per patient (IQR: 1-4), 64\% of which were hydralazine. The majority (380 [64\%]) were ordered on an "as needed" basis, while 217 (36\%) were administered as a onetime dose. Three-quarters of all doses were ordered by the teaching service (456 [76\%]), with the remaining $24 \%$ ordered by the direct-care (hospitalist) service.

During the baseline period among patients receiving IV antihypertensive medications, the median SBP of the population prior to treatment was $187 \mathrm{~mm} \mathrm{Hg}$ (IQR 177-199; Table 2). Treatment was initiated in $30 \%$ of patients for an SBP $<180$ $\mathrm{mm} \mathrm{Hg}$ and in $75 \%$ for an SBP $<200 \mathrm{~mm} \mathrm{Hg}$. The median time to follow-up BP check was 34 minutes (IQR 15-58). The median decrement in SBP was $20 \mathrm{~mm} \mathrm{Hg}$ (IQR 5-37); however, the response to treatment was highly variable, with $2 \%$ of patients experiencing no change and $14 \%$ experiencing an increase in SBP. Seventy-nine patients (14\%) had a decrement in SBP $>25 \%$ following treatment. 
TABLE 2. Patient-Level Logistic Regression Analysis of the Association between Receipt of Intravenous Antihypertensive Medication and Exposure to $\mathrm{Ol}$ Intervention ${ }^{\mathrm{a}}$

\begin{tabular}{|c|c|c|}
\hline Variable & $\begin{array}{l}\text { Odds of Treatment } \\
\qquad(95 \% \mathrm{Cl})\end{array}$ & $P$ Value \\
\hline Postintervention period & $0.62(0.47-0.83)$ & .001 \\
\hline \multicolumn{3}{|l|}{ Race } \\
\hline White & ref. & ref. \\
\hline Asian & $1.33(0.92-1.93)$ & .13 \\
\hline Hispanic & $1.49(0.96-2.20)$ & .08 \\
\hline Black & $1.81(1.29-2.53)$ & .001 \\
\hline Other/unknown & $1.38(0.86-2.20)$ & .18 \\
\hline \multicolumn{3}{|l|}{ Age } \\
\hline $18-53$ & ref. & ref. \\
\hline $54-66$ & $0.68(0.48-0.97)$ & .03 \\
\hline $67-77$ & $0.55(0.35-0.84)$ & .01 \\
\hline $78-116$ & $0.80(0.51-1.24)$ & .32 \\
\hline \multicolumn{3}{|l|}{ Sex } \\
\hline Male & ref. & ref. \\
\hline Female & $1.10(0.86-1.40)$ & .46 \\
\hline \multicolumn{3}{|l|}{ Language } \\
\hline English & ref. & ref. \\
\hline Non-English & $0.98(0.68-1.40)$ & .90 \\
\hline \multicolumn{3}{|l|}{ Insurance } \\
\hline Commercial & ref. & ref. \\
\hline Medicaid & $0.97(0.63-1.51)$ & .89 \\
\hline Medicare & $1.26(0.81-1.95)$ & .31 \\
\hline Other & $0.34(0.04-2.82)$ & .32 \\
\hline Preexisting hypertension & $4.25(2.75-6.56)$ & $<.001$ \\
\hline APR-DRG weight & $1.13(1.07-1.20)$ & $<.001$ \\
\hline Inpatient length of stay & $1.02(1.01-1.03)$ & $<.001$ \\
\hline
\end{tabular}

axposure dichotomized to pre- and postintervention time periods. Model adjusts for age, sex, race, ethnicity, primary language, insurance status, preexisting HTN, length of stay, and APR-DRG weight.

Abbreviation: APR-DRG, all patient refined diagnosis related-groups; HTN, hypertension.

Description of Quality Improvement Results

Following the $\mathrm{Ql}$ initiative, a total of 934 patients experienced 9,743 episodes of asymptomatic elevated blood pressure over a 4-month period (November 1, 2017 to February 28, 2018). As shown in Table 1, patients in the postintervention period had a slightly higher median age (67 [IOR 55-80] vs 69 [IQR 57-83]; $P=$ $.01)$, a higher median APR-DRG weight (1.34 [IQR 0.99-1.77] vs 1.48 [1.00-1.82]; $P<.001)$, and a longer median length of stay (4.6 [2.8-8.0] days vs 5.1 [2.9-9.2] days; $P=.004$ ). There was also a higher proportion of nonEnglish speakers, fewer Black patients, and a lower proportion of preexisting HTN, in the postintervention period.

Of the 934 patients with $\geq 1$ episode of asymptomatic elevated $\mathrm{BP}, 70(7 \%)$ were treated with IV antihypertensive medications, with a total of 196 doses administered. The proportion of patients treated per month during the postintervention period ranged from $6 \%$ to $8 \%$, which was the lowest of the entire study period and below the baseline average of 10\% (Figure).

In a patient-level logistic regression pre-post analysis adjusting for age, sex, race, ethnicity, primary language, insurance status, preexisting HTN, length of stay, and APR-DRG weight, patients admitted to the general medicine service during the postintervention period had 38\% lower odds of receiving IV antihypertensive medications than those admitted during the baseline period ( $\mathrm{OR}=0.62 ; 95 \% \mathrm{Cl} 0.47-0.83 ; P=.001)$. In this adjusted model, the following factors were independently associated with increased odds of receiving treatment: APR-DRG weight (OR 1.13; 95\% Cl 1.07-1.20; $P<.001)$, Black race (OR $1.81 ; 95 \% \mathrm{Cl} 1.29-2.53 ; P=.001)$, length of stay (OR 1.02; $95 \%$ $\mathrm{Cl} 1.01-1.03 ; P<.001)$, and preexisting HTN (OR 4.25; $95 \% \mathrm{Cl}$ 2.75-6.56; $P<.001)$. Older age was associated with lower odds of treatment (Table 2).

Among patients who received treatment, there were no differences between pre- and postintervention periods in the proportion of pretreatment SBP $<180 \mathrm{~mm} \mathrm{Hg}(29 \%$ vs $32 \%$; $P$ $=.40), 180-199 \mathrm{~mm} \mathrm{Hg}(47 \%$ vs $40 \% ; P=.10)$, or $>200 \mathrm{~mm} \mathrm{Hg}$ (25\% vs $28 \% ; P=.31$; Table 3 ).

Population-level median SBP was similar between pre- and postintervention periods ( $167 \mathrm{~mm} \mathrm{Hg}$ vs $168 \mathrm{~mm} \mathrm{Hg}, P=.78$ ), as were unadjusted rates of rapid response calls, ICU transfers, and code blues (Table 3). After adjustment for baseline characteristics and illness severity at the patient level, the odds of rapid response calls (OR 0.84; 95\% $\mathrm{Cl} 0.65-1.10 ; P=.21$ ) and ICU transfers (OR 1.01; 95\% Cl 0.75-1.38; $P=.93$ ) did not differ between pre- and postintervention periods. A regression model was not fit for cardiopulmonary arrests due to the low absolute number of events.

\section{CONCLUSIONS}

Our results suggest that treatment of asymptomatic elevated BP using IV antihypertensive medications is common practice at our institution. We found that treatment is often initiated for only modestly elevated BPs and that the clinical response to these medications is highly variable. In the baseline period, one in seven patients experienced a decrement in BP $>25 \%$ following treatment, which could potentially cause harm. ${ }^{11}$ There is no evidence, neither are there any consensus guidelines, to support the rapid reduction of BP among asymptomatic patients, making this a potential valuable opportunity for reducing unnecessary treatment, minimizing waste, and avoiding harm.

While there are a few previously published studies with similar results, we add to the existing literature by studying a larger population of more than 3,000 total patients, which was uniquely multiracial, including a high proportion of non-English speakers. Furthermore, our cohort included patients in the ICU, which is reflected in the higher-than-average APRDRG weights. Despite being critically ill, these patients arguably still do not warrant aggressive treatment of elevated BP when asymptomatic. By excluding symptomatic BP elevations using surrogate markers for end-organ damage in addition to discharge diagnosis codes indicative of conditions in which 
TABLE 3. Treatment Characteristics, Response to Treatment, and Outcomes Compared between Pre- and Postintervention Periods

\begin{tabular}{|c|c|c|c|}
\hline & Baseline Period & Postintervention & \\
\hline Treatment Characteristics & $n=597$ doses & $\mathrm{n}=196$ doses & $P$ Value \\
\hline Hydralazine & $380(64 \%)$ & $97(50 \%)$ & \multirow[b]{2}{*}{$<.001$} \\
\hline Labetalol & $217(36 \%)$ & $99(50 \%)$ & \\
\hline \multicolumn{4}{|l|}{ Hour of medication administration, $n(\%)$} \\
\hline \multicolumn{4}{|l|}{ Pre-treatment SBP, n (\%) } \\
\hline$<180 \mathrm{mmHg}$ & $170(29 \%)$ & $62(32 \%)$ & .40 \\
\hline $180-199 \mathrm{mmHg}$ & $281(47 \%)$ & $79(40 \%)$ & .10 \\
\hline$>200 \mathrm{mmHg}$ & $146(24 \%)$ & $55(28 \%)$ & .31 \\
\hline \multicolumn{4}{|l|}{ Median SBP, mmHg (IQR) } \\
\hline Post-treatment & $165(150-185)$ & $171(154-186)$ & .18 \\
\hline \multicolumn{4}{|c|}{ Magnitude of blood pressure decrease, $n(\%)$} \\
\hline SBP decreased $<10 \%$ & $186(48 \%)$ & $61(41 \%)$ & .99 \\
\hline SBP decreased $10-25 \%$ & $223(38 \%)$ & $67(45 \%)$ & .42 \\
\hline SBP decreased $>25 \%$ & $79(14 \%)$ & $20(14 \%)$ & .27 \\
\hline Outcomes & $\mathrm{n}=2,306$ patients & $\mathrm{n}=934$ patients & \\
\hline Receipt of IV antihypertensive, $n(\%)$ & $251(11 \%)$ & $70(7 \%)$ & .003 \\
\hline \multicolumn{4}{|l|}{ Balancing outcomes, n (\%) } \\
\hline Rapid response calls & $294(12 \%)$ & $114(11 \%)$ & .72 \\
\hline
\end{tabular}

Abbreviations: ICU, intensive care unit; IV, intravenous; SBP, systolic blood pressure.

tight BP control may be warranted, we were able to study a more critically ill patient population. We were also able to describe which baseline patient characteristics convey higher adjusted odds of receiving treatment, such as preexisting HTN, younger age, illness severity, and black race.

Perhaps most significantly, our study is the first to demonstrate an effective $\mathrm{Q}$ I intervention aimed at reducing unnecessary utilization of IV antihypertensives. We found that this can feasibly be accomplished through a combination of educational efforts and systems changes, which could easily be replicated at other institutions. While the absolute reduction in the number of patients receiving treatment was modest, if these findings were to be widely accepted and resulted in a wide-spread change in culture, there would be a potential for greater impact.

Despite the reduction in the proportion of patients receiving IV antihypertensive medications, we found no change in the median SBP compared with the baseline period, which seems to support that the intervention was well tolerated. We also found no difference in the number of ICU transfers, rapid response calls, and cardiopulmonary arrests between groups.
While these findings are both reassuring, it is impossible to draw definitive conclusions about safety given the small absolute number of patients having received treatment in each group. Fortunately, current guidelines and literature support the safety of such an intervention, as there is no existing evidence to suggest that failing to rapidly lower BP among asymptomatic patients is potentially harmful. ${ }^{11}$

There are several limitations to our study. First, by utilizing a large electronic dataset, the quality of our analyses was reliant on the accuracy of the recorded EHR data. Second, in the absence of a controlled trial or control group, we cannot say definitively that our Ql initiative was the direct cause of the improved rates of IV antihypertensive utilization, though the effect did persist after adjusting for baseline characteristics in patient-level models. Third, our follow-up period was relatively short, with fewer than half as many patients as in the preintervention period. This is an important limitation, since the impact of $\mathrm{Q}$ interventions often diminishes over time. We plan to continually monitor IV antihypertensive use, feed those data back to our group, and revitalize educational efforts should rates begin to rise. Fourth, we were unable to directly measure 
which patients had true end-organ injury and instead used orders placed around the time of medication administration as a surrogate marker. While this is an imperfect measure, we feel that in cases where a provider was concerned enough to even test for end-organ injury, the use of IV antihypertensives was likely justified and was therefore appropriately excluded from the analysis. Lastly, we were limited in our ability to describe associations with true clinical outcomes, such as stroke or myocardial infarction, which could theoretically be propagated by either the use or the avoidance of IV antihypertensive medications. Fortunately, based on clinical guidelines and existing evidence, there is no reason to believe that reducing IV antihypertensive use would result in increased rates of these outcomes.

Our study reaffirms the fact that overutilization of IV anti-

\section{References}

1. Axon RN, Cousineau L, Egan BM. Prevalence and management of hypertension in the inpatient setting: A systematic review. J Hosp Med. 2011;6(7):417422. doi: 10.1002/jhm.804.

2. Herzog $E$, Frankenberger $O$, Aziz $E$, et al. A novel pathway for the management of ypertension for hospitalized patients. Crit Pathw Cardiol. 2007;6(4):150-160. doi: 10.1097/HPC.0b013e318160c3a7.

3. James PA, Oparil S, Carter BL, et al. 2014 evidence-based guideline for the management of high blood pressure in adults: report from the panel members appointed to the eighth Joint National Committee (JNC 8). JAMA. 2014;311(5):507-520. doi: 10.1001/jama.2013.284427.

4. Weder AB. Treating acute hypertension in the hospital: A lacuna in the guidelines [editorial]. Hypertension. 2011;57(1):18-20.

5. Axon RN, Turner M, Buckley R. An update on inpatient hypertension management. Curr Cardiol Rep. 2015;17(11):94. doi: 10.1007/s11886-015-0648-y.

6. Marik PE, Rivera R. Hypertensive emergencies: an update. Curr Opin Crit Care. 2011;17(6):569-580. doi:10.1097/MCC.0b013e32834cd31d.

7. Cherney D, Straus S. Management of patients with hypertensive urgencies and emergencies: a systematic review of the literature. J Gen Intern Med. 2002;17(12):937-945. doi: 10.1046/j.1525-1497.2002.20389.x.

8. Padilla Ramos A, Varon J. Current and newer agents for hypertensive emergencies. Curr Hypertens Rep. 2014;16(7):450. doi: 10.1007/s11906-0140450-z.

9. Whitworth JA, World Health Organization, International Society of Hypertension Writing Group. 2003 World Health Organization (WHO)/International Society of Hypertension (ISH) statement on management of hypertension. J Hypertens. 2003;21(11):1983-1992. doi: 10.1097/01.hjh.0000084751.37215.d2.

10. Campbell P, Baker WL, Bendel SD, White WB. Intravenous hydralazine for blood pressure management in the hospitalized patient: its use is of ten unjustified. J Am Soc Hypertens. 2011;5(6):473-477. doi: 10.1016/j. jash.2011.07.002. hypertensive medications among asymptomatic hospitalized patients is pervasive across hospital systems. This represents a potential target for a concerted change in culture, which we have demonstrated can be feasibly accomplished through education and systems changes.

Disclosures: Dr. Auerbach has current or pending grants from the CDC, PCORI and FDA that are unrelated to this research manuscript. He also receives royalties from UpToDate, and received an honorarium for being editor of JHM. Dr. Jacobs received a \$1,000 Resident/Fellow Travel Grant from the Society of Hospital Medicine to support the cost of travel to SHM, where he presented this research as a poster in 2018. Dr. Prasad receives money from EpiExcellence, LLC for consultation, which is unrelated to this research manuscript. All other authors have nothing to disclose.

11. Chobanian AV, Bakris GL, Black HR, et al. The Seventh Report of the Joint National Committee on Prevention, Detection, Evaluation, and Treatment of High Blood Pressure: the JNC 7 report. JAMA. 2003;289(19):2560-2572. doi: 10.1001/jama.289.19.2560.

12. Gauer R. Severe asymptomatic hypertension: Evaluation and treatment. Am Fam Physician. 2017;95(8):492-500

13. Lipari M, Moser LR, Petrovitch EA, Farber M, Flack JM. As-needed intravenous antihypertensive therapy and blood pressure control: Antihypertensive Therapy and BP Control. J Hosp Med. 2016;11(3):193-198. doi: 10.1002/jhm.2510.

14. Gaynor MF, Wright GC, Vondracek S. Retrospective review of the use of as-needed hydralazine and labetalol for the treatment of acute hypertension in hospitalized medicine patients. Ther Adv Cardiovasc Dis. 2017;12(1):7-15. doi: $10.1177 / 1753944717746613$

15. Weder $A B$, Erickson S. Treatment of hypertension in the inpatient setting: Use of intravenous labetalol and hydralazine. J Clin Hypertens. 2010;12(1):2933. doi: 10.1111/j.1751-7176.2009.00196.x.

16. Averill RF, Goldfield N, Hughes, JS, et al. All Patient Refined Diagnosis Related Groups (APR-DRGs) Version 20.0: Methodology Overview. Clinical Research and Documentation Departments of $3 \mathrm{M}$ Health Information Systems, Wallingford, Connecticut and Murray, Utah, 2003. https://www.hcup-us.ahrq. gov/. Accessed February 19, 2018.

17. lezzoni LI, Ash AS, Shwartz M, Daley J, Hughes JS, Mackiernan YD. Predicting who dies depends on how severity is measured: Implications for evaluating patient outcomes. Ann Intern Med. 1995;123(10):763-770.

18. Romagnoli S, Ricci Z, Quattrone D, et al. Accuracy of invasive arterial pressure monitoring in cardiovascular patients: an observational study. Crit Care. 2014:18(6):644 doi: 10.1186/s13054-014-0644-4.

19. Shojania K, Grimshaw JM. Evidence-based quality improvement: The state of the science. Health Aff (Millwood). 2005;24(1):138-150. doi: 10.1377/ hlthaff.24.1.138 